and hazards they face. It is out of this enormous amount of occupational health experience that I present this paper. Finally, my strive to attain the highest competence in occupational health made me register for MPH (Occupational and Environmental health) in Tulane University School of Public health and Tropical Medicine. New Orleans, USA. This is costing me over US\$50,000.00. With this financial commitment already made, I would grately appreciate the requested waiver support to attend this all important ICOH 2018 Congress. My sharing of my over 10 years of occupational health experience in 5 different countries and 4 continents in the would add a rich flavour to the program.

\section{FACTORS INFLUENCING CONSENT FOR AUTOPSIES FOR COMPENSATION PURPOSES AMONGST SOUTH AFRICAN MINERS}

1,2Julian Mthombeni, ${ }^{2}$ Ntombizodwa Ndlovu, ${ }^{2}$ Sara Nieuwoudt, ${ }^{2,3}$ Jill Murray*. ${ }^{1}$ Biomedical
Technology, Faculty of Health Sciences, University of Johannesburg, Johannesburg, South
Africa; ${ }^{2}$ School of Public Health, University of the Witwatersrand, Johannesburg, South
Africa; ${ }^{3}$ National Institute for Occupational Health, National Health Laboratory Service,
Johannesburg, South Africa

10.1136/oemed-2018-ICOHabstracts.686

Introduction Statutory, autopsy-based compensation for occupational lung diseases for South African miners began 100 years ago. Cardio-respiratory organs are removed locally where miners die, regardless of the clinical cause of death and with written consent from a near relative. The organs are examined at the National Institute for Occupational Health in Johannesburg. Previous studies in many countries have shown that the process of obtaining consent is a major determinant of autopsy uptake.

Mines provide organ removal services for mineworkers who die while employed. We determined uptake and explored experiences and perceptions of personnel involved in obtaining autopsy consent.

Methods A sequential mixed-methods study was conducted. The proportion of miners who died and had an autopsy was determined for three mines (2009 to 2012). Fourteen in-depth interviews were conducted among personnel involved in obtaining autopsy consent in the three mines. Thematic content analysis was applied.

Results Average autopsy uptake was $34 \%$ in the platinum mine, and $12 \%$ and $86 \%$ in the two gold mines. Procedures for obtaining consent were similar. The categories of personnel involved in the process included human resource officers, nurses, prosectors and union officials.

Barriers to the offering of autopsies were the location of death (consent was less likely to be offered if deaths occurred far from the mines), lack of awareness and poor knowledge and interpretation of the law governing compensation, work load and distrust of the inefficient compensation system. Enablers included sensitivity in communication (empathy, confidentiality, language and culture) and involvement of union officials.

Discussion Autopsy uptake was variable across the mining population and was influenced by several individual, sociocultural and institutional factors. Training is required to ensure that personnel who request consent are knowledgeable about the processes and benefits of autopsy compensation, and that they use appropriate communication strategies when talking to families.

\section{OCCUPATIONAL HEALTH IN MINING: RISK AND ASSURANCE EXPERIENCES}

${ }^{1}$ Claudina MCA Nogueira* ${ }^{2}$ André van der Linde, ${ }^{3} \mathrm{P}$ Rudolph Botha. ${ }^{1}$ Project Manager University of Pretoria, Faculty of Health Sciences, Pretoria, South Africa; ${ }^{2}$ Occupational Hygiene Consultant (retired) - ex-Anglo American plc, Johannesburg, South Africa; ${ }^{3}$ Lead: Mine Closure - Anglo American plc, Johannesburg, South Africa

\subsection{6/oemed-2018-ICOHabstracts.687}

Introduction Mineral extractive and processing industries are faced with difficult challenges regarding the management and control of risks inherent to the typical activities undertaken, with numerous hazards posing potential and serious risks to the safety, health and wellbeing of workforces and communities alike.

Methods Safety and health must be critical inclusion criteria across all stages of the mining lifecycle, i.e. exploration, design and construction, operation, extraction and processing, engineering and maintenance, and beyond, into mine closure and rehabilitation. Additionally, safety and health must be considered in transportation networks and distribution facilities, and in the manufacturing, recycling and disposal of mining products.

Occupational Health is driven by two disciplines - Occupational Hygiene and Occupational Medicine. These two pillars work together and complement each other through a process of Health Risk Assessment (HRA) and management.

Result In the workplace environment, the occupational HRA is the pivotal multi-disciplinary component to risk assessment, and a key consideration in the decision-making process.

The occupational HRA is the systematic identification and analysis of workplace hazards with the aim of implementing and monitoring control measures, i.e. actions and interventions that aid in eliminating or reducing the levels of hazardous exposures. These control measures apply across the board, and follow a Hierarchy of Control approach, from equipment, techniques, and processes; to protocols, policies and guidelines; as well as the education of workforces and communities living in areas surrounding mining operations.

Discussion The success of mining projects and operations hinges, among other criteria, on a sound understanding of the potential health risks for the development of inherently safer designs and operating systems.

This paper discusses learnings with respect to the occupational HRA requirements for mining operations and projects, in order to identify and mitigate the risks in terms of occupational health. Considerations for risk assessment methods are presented, together with the importance of ongoing review and quality assurance, to improve the HRA process.

\section{MERCURY FREE GOLD MINING: ADVANCING THE GLOBAL MINAMATA RESOLUTION}

${ }^{1}$ Erik Jørs*, ${ }^{2}$ Florencia Harari. 'Department of Occupational and Environmental Medicine, Odense University Hospital, Odense, Denmark; ${ }^{2}$ Department of Occupational and Environmental Medicine, Bispebjerg University Hospital, Copenhagen, Denmark

\subsection{6/oemed-2018-ICOHabstracts.688}

Aim of special session To discuss the toxicity of mercury versus borax, health affection of miners and legal aspects of mercury use in gold-mining.

Presenters: ${ }^{1}$ Jesper Bælum, ${ }^{2}$ Stephan Bose-O’Reilly, ${ }^{3}$ Florencia Harari, ${ }^{4}$ Jane F Thomsen, ${ }^{5}$ Jinky L Lu, ${ }^{6}$ Sophia F Lu 